\title{
Recommendations for a Standardized Report for Adult Transthoracic Echocardiography: A Report from the American Society of Echocardiography's Nomenclature and Standards Committee and Task Force for a Standardized Echocardiography Report
}

\author{
Julius M. Gardin, MD, David B. Adams, RDCS, Pamela S. Douglas, MD, Harvey \\ Feigenbaum, MD, David H. Forst, MD, Alan G. Fraser, MD, Paul A. Grayburn, MD, Alan \\ S. Katz, MD, Andrew M. Keller, MD, Richard E. Kerber, MD, Bijoy K. Khandheria, MD, \\ Allan L. Klein, MD, Roberto M. Lang, MD, Luc A. Pierard, MD, Miguel A. Quinones, MD, \\ and Ingela Schnittger, MD, Raleigh, North Carolina
}

\begin{abstract}
$\mathbf{T}$ The American Society of Echocardiography has published guidelines relating to standards for training (and certification), performance, nomenclature and measurement, and quality improvement related to echocardiography. ${ }^{1-15}$ However, the Society has not previously made recommendations about what constitutes the core variables, ie, measurements and other elements, to be included in a transthoracic echocardiographic report. A document addressing this topic would be timely and could serve the following purposes:(1) Promote quality by defining the basic core of measurements and statements that constitute the report; (2) encourage the comparison of serial echocardiograms performed in patients at the same site or different sites; (3) improve communication by expediting development of structured report form software; and (4) facilitate multicenter research and analyses of cost-effectiveness. Because of these considerations, Dr Richard Kerber, President of the American Society of Echocardiography, appointed a task force in 1998 to develop recommendations for a standardized report for adult echocardiography.Various international societies have recently addressed this issue and recommended standards.

The purpose of this report is not to define bow to record a proper adult cardiac ultrasound examination or how to perform the measurements but, rather, to serve as a guideline to what measure-
\end{abstract}

Reprint requests: American Society of Echocardiography, 1500 Sunday Dr, Suite 102, Raleigh, NC 27607.

J Am Soc Echocardiogr 2002;15:275-90.

Copyright 2002 by the American Society of Echocardiography. 0894-7317/2002/\$35.00+0 27/1/121536

doi: $10.1067 / \mathrm{mje} .2002 .121536$ ments and descriptive items should be included. It is the hope of the Task Force that publication of this document will further our ongoing efforts to improve the overall quality of the practice of echocardiography.

\section{REPORT FORMAT}

The adult transthoracic echocardiography report should be comprised of the following sections: (1) demographic and other identifying information, (2) echocardiographic (Doppler, if indicated) evaluation, and (3) summary. These sections will be outlined briefly below.

The Task Force believes that there must be some latitude regarding the precise format of the echocardiography report so long as it contains this basic information. For example, some laboratories may wish to use a graphic display of left ventricular regional wall motion, whereas others may choose a text format for reporting this information. When possible, this information should be coded in database format to facilitate retrieval and communication.

Ideally, when Doppler descriptive information is included, it should be integrated with the corresponding statements derived from echocardiographic images. For example, descriptive statements regarding a regurgitant prosthetic mitral valve derived from both echocardiographic imaging and Doppler examinations should be grouped together. However, some laboratories may prefer to group all the Doppler data under a separate section of the report. This is acceptable as long as the pertinent information is available. 


\section{Demographic and Other Identifying Information}

It is suggested that the echocardiography report include the following demographic and other identifying information: (1) patient's name and/or other unique identifier, (2) age, (3) gender, (4) indications for test, (5) height, (6) weight, (7) blood pressure (if available), (8) referring physician identification, (9) interpreting physician identification, and (10) date on which study was performed. Other identifying information that may be helpful include: (1) echocardiographic study media location (eg, disk or tape number); (2) date on which the study was ordered, read, transcribed (if applicable), and verified; (3) location of the patient (eg, outpatient, inpatient); (4) location where study was performed; (5) name or identifying information for person(s) performing the study (eg, sonographer, physician); (6) echocardiographic instrument identification; and (7) imaging views obtained or not obtained, especially if the study is suboptimal.

\section{Echocardiographic and Doppler Evaluation}

\section{CARDIAC STRUCTURES}

The following cardiac and vascular structures are generally evaluated as part of a comprehensive adult transthoracic echocardiography report:

1. Left ventricle

2. Left atrium

3. Right atrium

4. Right ventricle

5. Aortic valve

6. Mitral valve

7. Tricuspid valve

8. Pulmonic valve

9. Pericardium

10. Aorta

11. Pulmonary artery

12. Inferior vena cava and pulmonary veins

It should be emphasized that identification and measurement of some of the structures listed may not always be possible or necessary to provide a comprehensive, clinically relevant report. However, it is important for the echocardiography report to include comments on the left ventricle, left atrium, mitral valve, and aortic valve. When images of these structures cannot be recorded or interpreted, the report should state that imaging was suboptimal or impossible. In addition, the indication for a particular echocardiographic study may make it crucial to image a particular anatomic structure or to obtain specific Doppler recording(s). In this case, it is important for the report to comment on the crucial findings or to note that an adequate recording was not possible.

\section{MEASUREMENTS}

As a general rule, quantitative measurements are preferable. However, it is recognized that qualitative or semiquantitative assessments are often performed and frequently adequate. The following types of measurements are commonly included in a comprehensive echocardiography report.

1. Left ventricle:

a. Size: Dimensions or volumes, at end-systole and end-diastole

b. Wall thickness and/or mass: Ventricular septum and left ventricular posterior wall thicknesses (at end-systole and end-diastole) and/or mass (at end-diastole)

c. Function: Assessment of systolic function and regional wall motion. Assessment of diastolic function

2. Left atrium:

- Size:Area or dimension

3. Aortic root:

- Dimension

4. Valvular stenosis:

a. For valvular stenosis: Assessment of severity. Measurements that provide an accurate assessment of severity include transvalvular gradient and area.

b. For subvalvular stenosis: Assessment of severity. Measurement of subvalvular gradient provides the most accurate assessment of severity and is therefore recommended.

5. Valvular regurgitation: Assessment of severity with semiquantitative descriptive statements and/or quantitative measurements

6. Prosthetic valves:

a. Transvalvular gradient and effective orifice area

b. Description of regurgitation, if present

7. Cardiac shunts:Assessment of severity. Measurements of $Q_{p}: Q_{S}$ (pulmonary-to-systemic flow ratio) and/or orifice area or diameter of the defect are often helpful.

DESCRIPTIVE STATEMENTS

Descriptive statements that echocardiographers may choose to use to describe pertinent findings are listed in the Appendix. These descriptive statements are broad in scope, but not all-inclusive, and are provided as an illustrative guide. Since these statements represent the universe of possible findings, they are not intended to be included in any single report. Rather, a carefully selected subset of these statements should be included in each report, balancing the needs for conciseness and completeness in responding to the patient's and referring physician's needs. Laboratories may choose to adapt these brief 
statements to construct sentences for use in this section of the report or in the Summary.

\section{SUMMARY}

A summary of the echocardiographic report often includes statements that: (1) Answer the question(s) posed by the referring physician, (2) emphasize abnormal findings, and (3) compare important differences and similarities of the current study versus previous echocardiographic studies, or reports, if available and deemed clinically relevant.

\section{APPENDIX}

The entire document, along with an Appendix including 12 subsections of descriptive statements, can be accessed at the web site of the American Society of Echocardiography, www.asecho.org.

\section{REFERENCES}

1. Shanewise JS, Cheung AT, Aronson S, et al. ASE/SCA guidelines for performing a comprehensive intraoperative multiplane transesophageal echocardiography examination: recommendations of the American Society of Echocardiography Council for Intraoperative Echocardiography and the Society of Cardiovascular Anesthesiologists Task Force for Certification in Perioperative Transesophageal Echocardiography. J Am Soc Echocardiogr 1999;12:884-900.

2. Ehler D, Carney DK, Dempsey AL, et al. Guidelines for cardiac sonographer education: report of the American Society of Echocardiography Sonographer Education and Training Committee. J Am Soc Echocardiogr 2001;14:77-84.

3. Pearlman AS, Gardin JM, Martin RP, et al. Guidelines for physician training in transesophageal echocardiography: recommendations of the American Society of Echocardiography Committee for Physician Training in Echocardiography. J Am Soc Echocardiogr 1992;5:187-94.
4. Pearlman AS, Gardin JM, Martin RP, et al. Guidelines for optimal physician training in echocardiography: recommendations of the American Society of Echocardiography Committee for Physician Training in Echocardiography. J Am Cardiol 1987;60:158-63.

5. Meyer RA, Hagler D, Huhta J, et al. Guidelines for physician training in pediatric echocardiography: recommendations of the Society for Pediatric Echocardiography Committee on Physician Training. J Am Cardiol 1987;60:164-5.

6. Bommer WJ, Shah P, Allen H, et al. The safety of contrast echocardiography: report of the committee on contrast echocardiography for the American Society of Echocardiography. JAm Coll Echo 1984;3:6-13.

7. Popp R, Agatston A, Armstrong W, et al. Recommendations for training in performance and interpretation of stress echocardiography. J Am Soc Echocardiogr 1998;11:95-6.

8. Armstrong WF, Pellikka PA, Ryan T, et al. Stress echocardiography: recommendations for performance and interpretation of stress echocardiography. J Am Soc Echocardiogr 1998;11:97-104.

9. Schiller NB, Maurer G, Ritter SB, et al. Transesophageal echocardiography. J Am Soc Echocardiogr 1989;2:354-7.

10. Schiller NB, Shah PM, Crawford M, et al. Recommendations for quantitation of the left ventricle by two-dimensional echocardiography. J Am Soc Echocardiogr 1989;2:358-67.

11. Henry WL, DeMaria A, Gramiak R, et al. Report of the American Society of Echocardiography Committee on Nomenclature and Standards in two-dimensional echocardiography. Circulation 1980;62:212-5.

12. Henry WL, DeMaria A, Feigenbaum H, et al. Report of the American Society of Echocardiography Committee on Nomenclature and Standards: identification of myocardial wall segments. November 1982:1-15.

13. Silverman NH, Sahn DJ, Allen HD. Society of Pediatric Echocardiography/American Society of Echocardiography: suggested nomenclature for cardiac septa. Addendum to Report of the American Society of Echocardiography Committee of Nomenclature and Standards: identification of myocardial wall segments. January 1986:1-4.

14. Sahn DJ, Baker DW, DeMaria A, et al. Recommendations for terminology and display for Doppler echocardiography. August 1984:1-11.

15. Kisslo J, Byrd BF III, Geiser EA, et al. Recommendations for continuous quality improvement in echocardiography. J Am Soc Echocardiogr 1995;8(Part 2):S1-28. 


\section{APPENDIX}

\section{LEFT VENTRICLE}

A. Cavity size (dimensions or volumes)

1. Normal

2. Increased
a. Mild
b. Moderate
c. Severe

3. Decreased

B. Wall thickness

1. Normal

2. Increased
a. Mild
b. Moderate
c. Severe

3. Decreased

4. Relative wall thickness

C. Ventricular mass
1. Normal
2. Borderline
3. Increase
1. Normal
a. Mild
b. Moderate
c. Severe

4. Left ventricular (LV) mass (or LV mass index)

D. Ventricular shape

2. Concentric hypertrophy

3. Asymmetric hypertrophy

a. Absent

b. Present
1. Anterior
2. Posterior
3. Septal
4. Lateral
5. Apical
6. Basal

4. Eccentric hypertrophy
a. Absent
b. Present

5. Aneurysm

a. Absent

b. Present

1. Anterior

2. Posterior

3. Inferior

4. Septal

5. Lateral

6. Apical

7. Other (specify)

6. Pseudoaneurysm
a. Absent
b. Present
1. Anterior
2. Posterior

3. Inferior

4. Septal

5. Lateral

6. Apical

7. Basal

E. Systolic function

1. Global

a. Ejection fraction

1. Normal

2. Borderline

3. Low normal

4. Decreased

a. Mild

b. Mild-to-moderate

c. Moderate

d. Moderate-to-severe

e. Severe

5. Increased (hyperdynamic)

b. Fractional shortening (basal)

1. Normal

2. Decreased

3. Increased

c. Fractional area change

1. Normal

2. Decreased

3. Increased

d. Dilated (congestive)

1. Mild

2. Mild-to-moderate

3. Moderate

4. Moderate-to-severe

5. Severe

2. Regional

a. Norma

b. Hypokinetic $\square 16$ or 17 segment

c. Akinetic $\quad$ ASE model

d. Dyskinetic List score for each segment

e. Scar/thinning

f. Not seen

In the case of abnormal septal motion, may code

1. Abnormal (paradoxical) motion consistent with right ventricular (RV) volume overload and/or elevated RV end-diastolic pressure

2. Abnormal (paradoxical) motion consistent with postoperative status

3. Abnormal (paradoxical) motion consistent with left bundle branch block

4. Abnormal (paradoxical) motion consistent with RV pacemaker

5. Abnormal (paradoxical) motion due to pre-excitation

6. Flattened in diastole (" $D$ "-shaped left ventricle) consistent with RV volume overload 
7. Flattened in systole consistent with RV pressure overload

8. Flattened in systole and diastole consistent with RV pressure and volume overload

9. Septal "bounce" consistent with constrictive physiology

10. Excessive respiratory change-consider tamponade and ventilationrelated

11. Other (specify)

3. Myocardial infarction

a. Anterior

1. Small

2. Small-to-moderate

3. Moderate

4. Moderate-to-large

5. Large

b. Posterior

1. Small

2. Small-to-moderate

3. Moderate

4. Moderate-to-large

5. Large

c. Inferior

1. Small

2. Small-to-moderate

3. Moderate

4. Moderate-to-large

5. Large

d. Lateral

1. Small

2. Small-to-moderate

3. Moderate

4. Moderate-to-large

5. Large

e. Anteroseptal

1. Small

2. Small-to-moderate

3. Moderate

4. Moderate-to-large

5. Large

f. Apical

1. Small

2. Small-to-moderate

3. Moderate

4. Moderate-to-large

5. Large

g. Anteroapical

1. Small

2. Small-to-moderate

3. Moderate

4. Moderate-to-large

5. Large

h. Posterolateral

1. Small

2. Small-to-moderate
3. Moderate

4. Moderate-to-large

5. Large

i. Other (specify)

1. Small

2. Small-to-moderate

3. Moderate

4. Moderate-to-large

5. Large

F. Diastolic filling

1. Normal

- For age

2. Abnormal

a. Pattern

1. Impaired relaxation

2. "Pseudonormal"

3. Restrictive

b. Elevated LV filling pressure

1. Absent

2. Present

a. Elevated mean left atrial pressure (LAP)

b. Elevated LV end-diastolic pressure (LVEDP)

c. Both elevated LAP and LVEDP

G. Thrombus

1. Absent

2. Present

a. Size

1. Small

2. Moderate

3. Large

b. Location

1. Apical

2. Basal

3. Lateral

4. Septal

c. Shape

1. Flat (mural)

2. Protruding

3. Pedunculated

4. Spherical

5. Regular

6. Irregular

7. Multilobular

8. Other (specify)

d. Texture

1. Solid

2. Layered

3. Hypoechoic interior (cystic)

4. Hyperechoic

5. Calcified

e. Mobility

1. Mobile

2. Fixed (Sessile)

f. Dimensions

H. Mass (tumor) 
1. Absent

2. Present

a. Size

1. Small

2. Moderate

3. Large

b. Location

1. Apical

2. Basal

3. Septal

4. Lateral

5. Intramyocardial

6. Intracavitary

c. Shape

1. Flat (mural)

2. Protruding

3. Pedunculated

4. Papillary

5. Spherical

6. Regular

7. Irregular

8. Multilobular

9. Frondlike

10. Infiltrating

d. Texture

1. Solid

2. Layered

3. Hypoechoic interior (cystic)

4. Echogenic

5. Calcified

e. Mobility

1. Mobile

2. Fixed (sessile)

f. Dimensions

I. Ventricular septal defect

1. Absent

2. Present

a. Location

1. Membranous (infracristal)

2. Infundibular (supracristal)

3. Inlet

4. Muscular

5. Multiple

- Designate

b. Size

1. Small

2. Moderate

3. Large

4. Dimensions

c. Shunt

1. Left-to-right

2. Right-to-left

3. Bidirectional

4. Qp:Qs ratio

\section{LEFT ATRIUM}

A. Normal
B. Abnormal

1. Size
a. Normal
b. Dilated
c. Mildly dilated
d. Moderately dilated
e. Severely dilated
f. Elongated
g. Volume (or dimensions)

2. Thrombus

a. Absent

b. Present

1. Size
a. Small
b. Moderate
c. Large

2. Location

a. LA cavity

1. Superior

2. Inferior

3. Lateral

4. Atrial septum

b. LA appendage

3. Shape
a. Flat (mural)
b. Protruding
c. Pedunculated
d. Papillary
e. Spherical
f. Regular
g. Irregular
h. Multilobular
i. Other (specify)

4. Texture
a. Solid
b. Layered
c. Hypoechoic interior (cystic)
d. Echogenic
e. Calcified

5. Mobility

a. Mobile

b. Fixed (sessile)

6. Dimensions

3. Mass

a. Absent

b. Present

1. Size
a. Small
b. Moderate
c. Large

2. Location

a. LA cavity

b. LA appendage

c. Fossa ovalis/atrial septum

3. Attachment site

a. Fossa ovalis/atrial septum

b. Left atrial body 

c. Mitral valve
d. Other (specify)

4. Shape

a. Flat (mural)

b. Protruding

c. Pedunculated

d. Papillary

e. Spherical

f. Regular

g. Irregular

h. Multilobular

i. Frondlike

j. Other (specify)

5. Texture
a. Solid
b. Layered
c. Layered
d. Hypoechoic interior (cystic)
e. Echogenic
f. Calcified

6. Mobility
a. Mobile
b. Fixed (sessile)

7. Dimensions

8. Type

a. Suggestive of myxoma

b. Suggestive of papilloma

c. Suggestive of fibroelastoma

d. Suggestive of other mass (specify)

4. Catheter

a. Absent

b. Present

1. LA cavity

2. LA appendage

5. Spontaneous echo contrast

a. Absent

b. Present

1. Degree

a. Mild

b. Severe

2. Location
a. LA cavity
b. LA appendage
c. LA cavity and LA appendage

6. Atrial septal defect

a. Location

1. Primum

2. Secundum

3. Sinus Venosus

b. Size

1. Small

2. Moderate

3. Large

c. Shunt

1. Left-to-right

2. Right-to-left

3. Bidirectional
4. Qp:Qs ratio

7. Patent foramen ovale

a. Absent

b. Present

1. Left-to-right shunt

2. Right-to-left shunt

3. Bidirectional shunt

8. Other

a. Cor triatriatum

b. Hypoplastic left atrium

c. Appearance consistent with cardiac transplantation

\section{RIGHT ATRIUM}

A. Normal

B. Abnormal

1. Size
a. Normal
b. Mildly enlarged
c. Moderately enlarged
d. Markedly enlarged
e. Small

2. Thrombus

a. Absent

b. Present

1. Size
a. Small
b. Moderate
c. Large

2. Location

a. RA cavity

b. RA appendage

c. Extending from inferior vena cava

3. Shape
a. Flat (mural)
b. Protruding
c. Pedunculated
d. Papillary
e. Spherical
f. Regular
g. Irregular
h. Multilobular
i Other (specify)

4. Texture
a. Solid
b. Layered
c. Hypoechoic interior (cystic)
d. Echogenic

e. Calcified

f. Other (specify)

5. Mobility

a. Mobile

b. Fixed (sessile)

6. Dimensions

3. Mass
a. Absent
b. Present 
1. Size
a. Small
b. Moderate
c. Large

2. Location

a. RA cavity

b. RA appendage

c. Extending from inferior vena cava

3. Shape

a. Flat (mural)

b. Protruding

c. Pedunculated

d. Papillary

e. Spherical

f. Regular

g. Irregular

h. Multilobular

i. Frondlike

j. Other (specify)

4. Texture

a. Solid

b. Layered

c. Hypoechoic interior (cystic)

d. Echogenic

e. Calcified

5. Mobility

a. Mobile

b. Fixed (sessile)

6. Type

a. Suggestive of myxoma

b. Suggestive of papilloma

c. Suggestive of fibroelastoma

d. Suggestive of other mass (specify)

7. Dimensions

4. Catheter/pacemaker wire

a. Absent

b. Present

1. RA cavity

2. RA appendage

5. Spontaneous contrast

a. Absent

b. Present

1. Degree

a. Mild

b. Severe

2. Persistence

a. Intermittent

b. Continuous

3. Location
a. RA cavity
b. RA appendage
c. RA cavity and RA appendage

6. Pressure

a. Interatrial septum bowed toward the left, consistent with elevated right atrial pressures

b. Dilated coronary sinus consistent with elevated right atrial pressures or a persistent left superior vena cava.

c. Dilated hepatic veins

d. Dilated inferior vena cava with poor inspiratory collapse consistent with elevated right atrial pressures

7. Other
a. Appropriate appearance for a tranplant recipient
b. Prominent eustachian valve
c. Prominent Chiari network

\section{RIGHT VENTRICLE}

A. Normal

B. Abnormal

1. Cavity size
a. Normal
b. Mildly enlarged
c. Moderately enlarged
d. Severely enlarged
e. Small

2. Wall thickness
a. Normal
b. Mildly increased
c. Moderately increased
d. Severely increased
e. Decreased
f. Thinned

3. Global systolic function
a. Normal
b. Hyperdynamic
c. Low normal
d. Mildly reduced
e. Moderately reduced
f. Severely reduced

4. Segmental wall analysis
a. Normal
b. Free wall
1. Hypokinetic
2. Akinetic
3. Dyskinetic

c. Septum

1. Abnormal (paradoxical) motion consistent with RV volume overload and/ or elevated RV end-diastolic pressure

2. Abnormal (paradoxical) motion consistent with postoperative status

3. Abnormal (paradoxical) motion consistent with left bundle branch block

4. Abnormal (paradoxical) motion consistent with RV pacemaker

5. Abnormal (paradoxical) motion due to pre-excitation

6. Flattened in diastole ("D"-shaped left ventricle) consistent with RV volume overload 
7. Flattened in systole consistent with RV pressure overload

8. Flattened in systole and diastole consistent with RV pressure and volume overload

9. Septal "bounce" consistent with constrictive physiology

10. Excessive respiratory change-consider tamponade and ventilation-related

11. Other (specify)

d. Apex

1. Hypokinetic

2. Akinetic

3. Dyskinetic

5. Miscellaneous

a. Consistent with cor pulmonale

b. Consistent with RV dysplasia

c. Consistent with RV infarction

\section{AORTIC VALVE}

A. Structure

1. Normal

2. Unicuspid

3. Bicuspid

- Specify raphe' location

4. Quadricuspid

5. Doming

6. Focal thickening

7. Diffuse thickening (sclerosis) without reduced excursion

8. Diffuse thickening with reduced excursion

9. Normally functioning mechanical prosthetic valve

a. Tilting disk

b. Bileaflet

c. Ball and cage

d. Other (specify)

e. Manufacturer

f. Size

10. Abnormally functioning mechanical prosthetic valve

a. Type

1. Tilting disk

2. Bileaflet

3. Ball and cage

4. Other (specify)

5. Manufacturer

6. Size

b. Abnormality (findings consistent with)

1. Rocking

2. Vegetation (see below)

3. Thrombus/mass

4. Dehiscence

5. Stenosis (see below)

6. Regurgitation (see below)

a. Physiologic

b. Prosthetic c. Peri-prosthetic

7. Abscess (see below)

8. Pannus

9. Fistula

- Describe

10. Fracture/perforation

- Describe

11. Normally functioning bioprosthetic valve

a. Porcine

b. Homograft

c. Pericardial

d. Native pulmonic

e. Size

f. Manufacturer

g. Other (specify)

12. Abnormally functioning bioprosthetic valve

a. Type

1. Porcine

2. Homograft

3. Pericardial

4. Native pulmonic

5. Size

6. Manufacturer

7. Other (specify)

b. Abnormality (findings consistent with)

1. Rocking/dehiscence

2. Vegetation (see below)

3. Thrombus/mass (see below)

4. Dehiscence

5. Stenosis (see below)

6. Regurgitation (see below)

a. Physiologic

b. Prosthetic

c. Periprosthetic

7. Abscess (see below)

8. Pannus

9. Fistula

- Describe

10. Fracture/perforation

- Describe

13. Vegetation

a. Location

1. Right coronary cusp

2. Noncoronary cusp

3. Left coronary cusp

4. Right coronary cusp and noncoronary cusp

5. Right coronary cusp and left coronary cusp

6. Noncoronary cusp and left coronary cusp

7. Right, noncoronary, and left coronary cusp

b. Mobility

1. Nonmobile

2. Mobile

3. Pedunculated and mobile 
c. Size

d. Other (specify)

14. Abscess

a. Location

1. Right coronary cusp/annulus

2. Left coronary cusp/annulus

3. Noncoronary cusp/annulus

b. Size
1. Small
2. Moderate
3. Large
4. Dimensions

15. Perforation

a. Right coronary cusp/annulus

b. Left coronary cusp/annulus

c. Noncoronary cusp/annulus

16. Mass

a. Location

1. Right coronary cusp

2. Left coronary cusp

3. Noncoronary cusp

b. Size

1. Small

2. Moderate

3. Large

4. Dimensions

17. Other (specify)

B. Function:Aortic stenosis

1. Severity
a. None
b. Mild
c. Mild-to-moderate
d. Moderate
e. Moderate-to-severe
f. Severe

2. Quantitative measurements

a. Peak aortic velocity (Doppler)

b. Peak instantaneous transaortic gradient (Doppler)

c. Peak LV outflow tract velocity

d. Mean aortic velocity (Doppler)

e. Mean transaortic gradient (Doppler)

f. LV outflow tract diameter

g. Aortic valve (effective orifice) area

h. Recording view from which peak aortic velocity obtained

1. Apical

2. Suprasternal

3. Right parasternal

4. Right supraclavicular

5. Other (specify)

C. Function:Aortic regurgitation

1. Severity
a. None
b. Trace (trivial)
c. Mild
d. Mild-to-moderate

e. Moderate

f. Moderate-to-severe

g. Severe

2. (Semi) Quantitative measurements

a. Pressure half-time

b. Regurgitant volume (or fraction)

c. Holodiastolic flow reversal present

1. In descending aorta

2. In abdominal aorta

\section{MITRAL VALVE}

A. Structure/function

1. Normal

2. Rheumatic

3. Myxomatous (redundant)

4. Prolapse

a. Anterior leaflet

1. Mild

2. Moderate

3. Severe

4. Medial segment

5. Middle segment

6. Lateral segment

7. Multiple segments

- Describe

b. Posterior leaflet

1. Mild

2. Moderate

3. Severe

4. Medial scallop

5. Middle scallop

6. Lateral scallop

7. Multiple scallops

- Describe

c. Holosystolic

d. Late systolic

e. Maximum degree of prolapse

5. Flail leaflet(s)

a. Anterior leaflet

1. Mild

2. Moderate

3. Severe

4. Medial segment

5. Middle segment

6. Lateral segment

7. Multiple segments

b. Posterior leaflet

1. Mild

2. Moderate

3. Severe

4. Medial scallop

5. Middle scallop

6. Lateral scallop

7. Multiple scallops

6. Ruptured chordae

a. Anterior leaflet

1. Mild 


\section{Moderate \\ 3. Severe}

b. Posterior leaflet

1. Mild

2. Moderate

3. Severe

4. Other

7. Other chordal disease
a. Shortening
b. Fusion
c. Other
d. Involving anterior leaflet
e. Involving posterior leaflet
f. Involving both leaflets

8. Leaflet elongation

a. Anterior leaflet

1. Mild

2. Moderate

3. Severe

b. Posterior leaflet

1. Mild

2. Moderate

3. Severe

9. Leaflet thickening/calcification

a. Anterior leaflet

1. Mild

2. Moderate

3. Severe

b. Posterior leaflet

1. Mild

2. Moderate

3. Severe

10. Leaflet mobility

a. Normal

b. Abnormal

1. Anterior mitral leaflet

a. Mildly decreased mobility

b. Moderately decreased mobility

c. Immobile

2. Posterior mitral leaflet
a. Mildly decreased mobility
b. Moderately decreased mobility
c. Immobile

11. Annular calcification
a. Mild
b. Moderate
c. Severe

12. Subvalvular disease

a. Calcification

1. Mild

2. Moderate

3. Severe

b. Thickening/fibrosis

1. Mild

2. Moderate

3. Severe

13. Annular dilatation
a. Mild
b. Moderate
c. Severe

14. Vegetation
a. Anterior leaflet
1. Nonmobile
2. Mobile
3. Pedunculated and mobile
4. Size
a. Small
b. Moderate
c. Large
d. Dimensions
5. Other (specify)

b. Posterior leaflet

1. Nonmobile

2. Mobile

3. Pedunculated and mobile

4. Size
a. Small
b. Moderate
c. Large
d. Dimensions

5. Other (specify)

15. Abscess
a. Location
1. Anterior leaflet/annulus
2. Posterior leaflet/annulus
3. Intravalvular (pars) fibrosa
b. Size
1. Small
2. Moderate
3. Large
4. Dimensions

16. Cleft
a. Anterior leaflet
1. Small
2. Moderate
3. Large
b. Posterior leaflet
1. Small
2. Moderate
3. Large

17. Systolic anterior motion
a. Anterior leaflet
1. Mild
2. Moderate
3. Severe
b. Posterior leaflet
1. Mild
2. Moderate
3. Severe
c. Chordal
1. Mild
2. Moderate
3. Severe

18. Dilated annulus 
- Dimensions

19. Increased E-point septal separation

20. Mitral presystolic closure

21. Interrupted A-C closure (B-notch)

22. Diastolic fluttering

a. Anterior leaflet

b. Posterior leaflet

23. Prosthetic Valve

a. Type

1. Mechanical
a. Tilting disk
b. Bileaflet
c. Ball and cage
d. Other (specify)
e. Manufacturer
f. Size

2. Bioprosthetic
a. Porcine
b. Homograft
c. Pericardial
d. Other (specify)
e. Manufacturer
f. Size

b. Abnormality (findings suggestive of)

1. Rocking

2. Dehiscence

3. Vegetation (see below)

4. Thrombus/mass

5. Stenosis (see below)

6. Regurgitation (see below)
a. Physiologic
b. Prosthetic
c. Periprosthetic

7. Pannus

8. Fistula

- Describe

9. Fracture/perforation

- Describe

24. Status-post mitral annular ring insertion

B. Mitral stenosis

1. Severity
a. None
b. Mild
c. Mild-to-moderate
d. Moderate
e. Moderate-to-severe
f. Severe

2. Quantitative measurements (if mitral stenosis present)

a. Mitral valve area

1. By planimetry

2. By Doppler pressure half-time

3. By other method (specify)

b. Mean transmitral velocity (Doppler)

c. Mean transmitral gradient (Doppler)

3. Status-post commissurotomy

a. Yes b. No

C. Mitral regurgitation

1. Severity

a. None

b. Mild

c. Mild-to-moderate

d. Moderate

e. Moderate-to-severe

f. Severe

2. Jet direction
a. Anteriorly directed
b. Posteriorly directed
c. Centrally directed
d. Wall-impinging jet
e. Impingement on pulmonary veins

3. Diastolic mitral regurgitation
a. Present
b. Absent

4. Quantitative measurements (if mitral regurgitation present)

a. Mitral regurgitation jet/left atrial area ratio

b. Pulmonary venous flow

1. Normal pulmonary venous flow

2. Blunted (decreased) systolic flow

3. Systolic flow reversal

c. Mitral regurgitant volume

1. By pulsed Doppler echo method

2. By PISA color Doppler method

d. Mitral regurgitant fraction

1. By pulsed Doppler echo method

2. By PISA color Doppler method

e. Effective mitral regurgitant orifice area

1. By pulsed Doppler echo method

2. By PISA color Doppler method

f. Vena contracta width

\section{TRICUSPID VALVE}

A. Structure

1. Normal

2. Abnormal

a. Rheumatic

b. Vegetation

1. Anterior leaflet

a. Nonmobile

b. Mobile

c. Pedunculated and mobile

d. Other (specify)

e. Size

(1) Small

(2) Moderate

(3) Large

(4) Dimensions

2. Posterior leaflet

a. Nonmobile

b. Mobile

c Pedunculated and mobile

d. Other (specify) 
e. Size
(1) Small
(2) Moderate
(3) Large
(4) Dimensions

3. Septal leaflet
a. Nonmobile
b. Mobile
c. Pedunculated and mobile
d. Other (specify)
e. Size
(1) Small
(2) Moderate
(3) Large
(4) Dimensions

c. Myxomatous (redundant)

d. Prolapse
1. Anterior leaflet
a. Mild
b. Moderate
c. Severe

2. Posterior leaflet
a. Mild
b. Moderate
c. Severe

3. Septal leaflet
a. Mild
b. Moderate
c. Severe
4. Holosystolic
5. Late systolic

e. Ruptured chordae/flail leaflet(s)

1. Anterior leaflet
a. Mild
b. Moderate
c. Severe

2. Posterior leaflet
a. Mild
b. Moderate
c. Severe

3. Septal leaflet
a. Mild
b. Moderate
c. Severe

f. Dilated annulus

- Dimensions

g. Ebstein's anomaly

h. Tricuspid atresia

B. Function: Regurgitation

1. Absent

2. Present

a. Severity

1. Trace

2. Mild

3. Mild-to-moderate

4. Moderate-to-severe

5. Severe b. Jet direction

1. Toward septum

2. Toward RA free wall

3. Central

4. Eccentric

5. Impinging on wall

6. Extending to dome (back wall of RA)

c. Hepatic vein systolic flow

1. Normal

2. Blunted (decreased)

3. Reversed

C. Function: Stenosis

1. Severity

a. None

b. Mild

c. Moderate

d. Severe

2. Quantitative measurements
a. Peak tricuspid velocity (Doppler)
b. Peak transtricuspid gradient (Doppler)
c. Mean tricuspid velocity (Doppler)
d. Mean transtricuspid gradient (Doppler)
e. Tricuspid valve area
f. Other (specify)

\section{PULMONIC VALVE}

A. Structure

1. Normal

2. Abnormal

a. Thickened

1. With good excursion

2. With mildly decreased excursion

3 . With moderately decreased excursion

4. With severely decreased excursion

b. Doming

c. Dilated annulus

d. Other (specify)

B. Function: Pulmonic regurgitation

1. Absent

2. Present
a. Mild
b. Mild-to-moderate
c. Moderate
d. Moderate-to-severe
e. Severe

C. Function: Pulmonic stenosis

1. Location

a. Valvular

b. Infundibular

c. Valvular and infundibular

d. Supravalvular

e. Branch

1. Left main pulmonary artery

2 . Right main pulmonary artery 3. Other (specify)

2. Severity

a. None 

b. Mild
c. Moderate
d. Severe

3. Quantitative measurements
a. Peak instantaneous pulmonary velocity (Doppler) b. Peak instantaneous transpulmonic gra-
dient (Doppler)
c. Mean pulmonary velocity (Doppler)
d. Mean transpulmonic gradient (Doppler)
e. Estimated pulmonary artery diastolic pressure
f. Other (specify)

D. Pulmonary pressure

1. PA systolic pressure

a. From tricuspid regurgitation jet

b. Other method (specify)

2. PA diastolic pressure (from pulmonary insufficiency jet)

3. Mean PA pressure (from pulmonary acceleration time)

\section{PERICARDIUM}

A. Normal

B. Abnormal

1. Effusion

a. Absent

b. Present

1. Size
a. Trivial
b. Small
c. Moderate
d. Large

2. Location

a. Generalized

b. Localized

(1) Near left ventricle

(2) Near right ventricle

(3) Near left atrium

(4) Near right atrium

3. Content
a. Fluid
b. Fibrinous
c. Focal strands
d. Effusive-constrictive

2. Thickening/Calcification

a. Absent

b. Present

3. Mass

a. Absent
b. Present

- Dimensions

4. Pleural effusion

a. Absent

b. Present

1. Right

2. Left
3. Right and left

5. Hemodynamic compromise

a. Tamponade

1. Absent

2. Present

b. Constriction

1. Absent

2. Present

c. Effuso-constrictive

1. Absent

2. Present

d. Septal bounce

1. Absent

2. Present

e. Inversion

1. Absent

2. Present
a. Left ventricle
b. Right ventricle
c. Left atrium
d. Right atrium

f. Excessive respiratory variation

1. Absent

2. Present

a. Ventricular dimensions

b. Mitral valve slope

c. Doppler flow velocities

(1) Mitral

(2) Tricuspid

(3) Aortic

(4) Pulmonic

(5) Hepatic

g. LV diastolic "checking" (square root sign)

1. Absent

2. Present

6. Fat Pad

a. Absent

b. Present

C. Ascites

1. Mild

2. Moderate

3. Severe

X. AORTA

A. Normal

B. Abnormal

1. Dilatation

a. Aortic root

b. Aortic root and the ascending aorta

c. Aortic root limited to the sinuses of Valsalva

d. Aortic root, ascending and descending aorta

e. Aortic root, sinuses of Valsalva and ascending aorta

f. Aortic root, ascending and transverse aorta

g. Aortic root, transverse, descending and ascending aorta 
h. Sinuses of Valsalva, aortic root, ascending and transverse aorta

i. Ascending aorta

j. Ascending and descending aorta

k. Ascending and transverse aorta

1. Ascending aorta and the sinuses of Valsalva

$\mathrm{m}$. Ascending, transverse and descending aorta

n. Descending aorta

2. Aneurysm

a. Location

1. Ascending aorta

2. Transverse aorta

3. Descending aorta

4. Ascending and transverse aorta

5. Ascending, transverse and descending aorta

6. Ascending and descending aorta

7. Transverse and descending aorta - Dimension(s) (maximum)

3. Plaque

a. Location

1. Ascending aorta

2. Transverse aorta

3. Descending aorta

4. Ascending and transverse aorta

5. Ascending and descending aorta

6. Ascending, transverse and descending aorta

7. Transverse and descending aorta

b. Characteristics

1. Layered

2. Protruding

3. Layered and protruding

4. Multilobular

5. Echolucent center

c. Size

1. Small

2. Moderate

3. Large

- Dimensions

d. Mobility

1. Mobile

2. Immobile

4. Graft

a. Type

1. Prosthetic

2. Homograft

b. Location

1. Ascending aorta

2. Ascending and transverse aorta

3. Descending aorta

4. Transverse aorta and descending aorta

5. Ascending, transverse, and descending aorta

5. Dissection

a. Location

1. Extending from the aortic root to the aortic arch
2. Extending from the aortic root to the ascending aorta

3. Extending from the aortic root to the descending aorta

4. Extending from the ascending aorta to the aortic arch

5. Extending from the ascending aorta to the descending aorta

6. Extending from the aortic arch to the descending aorta

7. Limited to the descending aorta

b. Entry site

1. Aortic root

2. Aortic arch

3. Ascending aorta

4. Descending aorta

c. Exit point

1. Ascending aorta

2. Aortic arch

3. Descending aorta

4. Multiple

- Describe

d. False lumen

1. Contains thrombus

2. Compressing the superior vena cava

3 . Compressing the true lumen

4. Contains thrombus and compressing the true lumen

e. Intramural hematoma

1. Ascending root

2. Extending from the aortic root to the ascending aorta

3. Extending from the aortic root to the descending aorta

4. Extending from the ascending aorta to the aortic arch

5. Extending from the ascending aorta to the descending aorta

6. Extending from the aortic arch to the descending aorta

7. Limited to the descending aorta

f. Classification

1. Stanford Type A (proximal)

2. Stanford Type B (distal)

3. DeBakey Type I

4. DeBakey Type II

5. DeBakey Type III

6. Other (specify)

6. Coarctation

a. Location

1. Proximal to left subclavian artery

2. Distal to left subclavian artery

b. Minimal diameter

c. Peak gradient

7. Transposition of the great arteries

8. Corrected transposition of the great arteries 


\section{PULMONARY ARTERY}

A. Normal

B. Dilated

1. Mildly dilated

2. Moderately dilated

3. Severely dilated

C. Suspect thromboembolism

1. Main pulmonary artery

2. Right pulmonary artery

3. Left pulmonary artery

D. Pulmonary hypertension

1. Absent

2. Present

E. Pulmonary artery hypoplasia

F. Pulmonary branch stenosis

1. Right pulmonary artery

2. Left pulmonary artery

G. Patent ductus arteriosus

H. Estimated pulmonary artery systolic pressure

\section{VENOUS}

A. Pulmonary veins

1. Not recorded

2. Normal

a. Left upper

b. Left lower

c. Right upper

d. Right lower

e. Left upper and left lower

f. Right upper and right lower

g. Left upper and right lower

h. Right upper and left lower

i. Three pulmonary veins

j. Four pulmonary veins

3. Abnormal

a. Size

1. Normal

2. Dilated

3. Stenotic

b. Location

1. Left upper

2. Left lower

3. Right upper

4. Right lower

5. Left upper and left lower

6. Right upper and right lower

7. Left upper and right lower

8. Right upper and left lower

9. Three pulmonary veins

10. Four pulmonary veins

c. Flow patterns (Doppler)

1. Normal

2. Systolic blunting

3. Systolic flow reversal (suggestive of severe MR)

4. Location of abnormal flow pattern

d. Thrombus

1. Absent
2. Present

e. Mass

1. Absent

2. Present

a. Location

(1) Left upper

(2) Left lower

(3) Right upper

(4) Right lower

(5) Left upper and left lower

(6) Right upper and right lower

(7) Left upper and right lower

(8) Right upper and left lower

(9) Three pulmonary veins

(10) Four pulmonary veins

b. Dimensions

f. Congenital anomalies

1. Left partial anomalous pulmonary venous return

2. Right partial anomalous pulmonary venous return

3. Pulmonary venous hypoplasia

B. Inferior vena cava (IVC)/hepatic vein

1. Not recorded

2. Normal

3. Abnormal

a. Size

1. Normal

2. Dilated

3. Stenotic

b. Respiratory change in dimension

1. $>50 \%$

2. $<50 \%$

3. $<$ Plethora

4. Dilated IVC with poor inspiration collapse consistent with elevated right atrial pressure

c. Estimated right atrial pressure

d. Flow patterns (Doppler)

1. Normal

2. Systolic blunting

3. Systolic flow reversal (suggestive of severe TR)

e. Thrombus

1. Absent

2. Present

- Dimensions

f. Mass

1. Absent

2. Present

- Dimensions

g. Pacer wire/venous catheter

1. Pacer wire

2. Venous catheter

h. Congenital anomaly

1. Azygous continuation of IVC to left SVC

2. Azygous continuation of IVC to right SVC 